\title{
Doctors' smoking control knowledge, attitudes and practices: a cross-sectional study conducted in Shandong Province, China
}

Qiang Wang ${ }^{1 \dagger}$, Xueli Zhang ${ }^{2 \dagger}$, Zengwu Wang ${ }^{3}$, Shangang Feng ${ }^{3}$, Yang $\mathrm{Li}^{4}$, Chuanfeng Zhang ${ }^{4}$ and Chunping Wang 5* $^{*}$

\begin{abstract}
Background: Doctors play an important role in smoking control. This study aimed to assess doctors' smoking control knowledge, attitudes and practices to help doctors raise awareness of smoking control assistance.

Methods: This cross-sectional study recruited 1046 doctors from Shandong Province, China, by using multistage sampling. Participants' information was collected by questionnaire. Pearson's $x^{2}$ test and Fisher's exact probability method were used to compare the distributions of categorical variables between/among groups.

Results: Among the participants, $14.7 \%$ were current smokers. Approximately $50.3 \%$ of participants had heard of smoking cessation drugs and $59.2 \%$ of participants thought that low-tar and low-nicotine cigarettes were as harmful to health as common cigarettes. Approximately 98.2 and $60.9 \%$ of participants agreed that smoking was related to lung cancer and male sexual dysfunction, respectively. Although $72.0 \%$ of participants believed that doctors should actively provide smoking cessation assistance, only $58.1 \%$ of participants considered that doctors should be responsible for providing smoking cessation assistance. Similarly, $85.2 \%$ of participants often asked about the smoking history of patients or their family members, while only $4.9 \%$ of participants had prescribed smoking cessation drugs for patients. Pediatricians had a higher proportion of "Agree" responses to the assessment items than doctors in other departments.
\end{abstract}

Conclusions: The results showed that doctors in Shandong Province did not have sufficient knowledge of smoking control. Slightly more than half of doctors thought that providing smoking cessation assistance was their responsibility. Only a few participants had prescribed smoking cessation drugs.

Keywords: Smoking control, Knowledge, Attitude, Practice

\footnotetext{
*Correspondence: chpwang@163.com

${ }^{\dagger}$ Qiang Wang and Xueli Zhang contributed equally to this work.

${ }^{5}$ Department of Environmental Health, Weifang Medical University, No. 7166 Baotong west street, Weifang 261053, Shandong, People's Republic of China

Full list of author information is available at the end of the article
}

(c) The Author(s). 2021 Open Access This article is licensed under a Creative Commons Attribution 4.0 International License, which permits use, sharing, adaptation, distribution and reproduction in any medium or format, as long as you give appropriate credit to the original author(s) and the source, provide a link to the Creative Commons licence, and indicate if changes were made. The images or other third party material in this article are included in the article's Creative Commons licence, unless indicated otherwise in a credit line to the material. If material is not included in the article's Creative Commons licence and your intended use is not permitted by statutory regulation or exceeds the permitted use, you will need to obtain permission directly from the copyright holder. To view a copy of this licence, visit http://creativecommons.org/licenses/by/4.0/. The Creative Commons Public Domain Dedication waiver (http://creativecommons.org/publicdomain/zero/1.0/) applies to the data made available in this article, unless otherwise stated in a credit line to the data. 


\section{Background}

Smoking is a preventable risk factor for many noncommunicable diseases [1]. Many interventions have been implemented to prevent smoking. However, the prevalence of smoking is still high, and the problems resulting from smoking are still serious. Worldwide, approximately $20 \%$ of people aged $\geq 15$ years were current smokers in 2015 [2]. Tobacco is responsible for seven million deaths annually [3]. In China, there are up to 300 million smokers [4]. According to the report of the Chinese Center for Disease Control and Prevention, $50.5 \%$ of males aged $\geq 15$ years in China were smokers in 2018 [5]. In addition, the rate of secondhand smoke exposure among nonsmoking people was $68.1 \%$ [5]. As a result, there were up to 1 million deaths related to smoking in China [6]. Thus, it is urgent to help smokers quit smoking, especially in China.

Counseling by doctors combined with pharmacotherapy is an effective smoking cessation intervention [7]. Simple and brief counseling by a doctor increased the probability of quitting smoking by $66 \%$ [8]. Smokers who were provided counseling and medication had a twofold higher likelihood of quitting smoking successfully than smokers who did not receive counseling and medication [9]. Doctors are regarded as role models for healthy lifestyles by patients [10]. In addition, doctors' counseling is more likely to be accepted by smoking patients due to doctors' specialized knowledge. Thus, doctors play a crucial role in providing counseling about smoking cessation [11]. Obviously, many studies have also proposed that doctors should theoretically provide counseling about smoking cessation for smokers in daily work [12]. However, doctors' responses to smoking control shown in previous studies are not satisfactory. A cross-sectional study conducted in China demonstrated that the harms of smoking were not well known by all doctors [10]. A cross-sectional study carried out in Egypt in 2011 showed that more than 50\% of doctors admitted that they had poor knowledge of smoking cessation interventions [9]. A qualitative study implemented in Armenia in 2015 reported that some doctors did not consider smoking cessation counseling to be their responsibility [7]. The 5As method (Ask: ask all patients about tobacco use; Advise: advise all tobacco users to quit smoking; Assess: assess the willingness to quit smoking; Assist: assist with quitting; Arrange: arrange follow-up) refers to one common strategy to address tobacco use and nicotine dependence [12]. A systematic literature review conducted in 2016 showed that $65 \%$ of doctors asked about their patients' smoking behavior, and $63 \%$ advised their patients to quit smoking [13]. In addition, this review reported that approximately 36, 44 and $22 \%$ of doctors assessed, assisted and arranged follow-up with their patients, respectively [13]. As the data demonstrated, the doctors' factual responses to smoking control do not match the doctors' expected responses. Thus, doctors' factual performance in smoking control should be reassessed and enhanced.

As China produces the largest number of cigarettes and has the highest number of smokers and tobaccorelated deaths [14], it is necessary to implement a smoke-free policy in China and help smokers quit smoking. The medical doctors' role in smoking control is particularly important. Shandong Province has the second largest population in China [15]. However, few studies have investigated the performance of doctors in smoking control in Shandong Province. In this study, we aimed to assess the factual representations of doctors registered in Shandong Province in smoking control based on their knowledge, attitudes and practices about smoking control, which may be able to fill the previous research gap and raise doctors' awareness of smoking control assistance.

\section{Methods \\ Definitions}

Smokers were defined as people who had smoked 100 or more cigarettes (or the equivalent amount of tobacco) during their lifetime [16]. Current smokers were defined as smokers who smoked any tobacco product at the time of the survey. Ex-smokers were defined as smokers who had not smoked at all in the last 3 months at the time of the survey. Never-smokers were defined as people who had smoked no more than 100 cigarettes (or the equivalent amount of tobacco) during their lifetime. Nonsmokers were defined as ex-smokers and never-smokers in this study.

\section{Sample size}

The formula used to calculate the sample size was as follows: $N=400 \times q \div p$ [17]. In this formula, $N$ is the sample size and $p$ is the incident rate of some event. $q=$ $1-p$. A previous study conducted in China showed that the prevalence of current smoking among doctors was $28.4 \%$ [18]. Therefore, we entered $p=28.4 \%$ into the formula and calculated the sample size to be 1008 . The last step of the sampling under study was cluster sampling. After the cluster sampling, the number of doctors included in this study was 1050. Therefore, the required sample size was changed to 1050 .

\section{Participants}

A cross-sectional study was conducted in Shandong Province, China, from July to December, 2018. Multistage sampling was carried out to select participants. Firstly, 17 cities of Shandong Province were divided into two groups according to social, cultural and geographical conditions. Secondly, one city was randomly sampled 
from each group as the sample city. Weifang and Liaocheng were selected. Thirdly, one 3-A-Class comprehensive hospital in Weifang and two 3-A-Class comprehensive hospitals in Liaocheng were randomly sampled as the sample hospitals, including Weifang People's Hospital, Liaocheng People's Hospital and Liaocheng Traditional Chinese Medicine Hospital. Fourthly, cluster sampling was conducted separately in each hospital to randomly sample departments. All doctors within the sampled departments were treated as participants and interviewed by the questionnaire used in the current study. This study was approved by the Ethics Review Committee of Weifang Medical University, and informed consent was obtained from all participants.

A total of 1050 doctors were included in this study, and 1046 valid questionnaires were retrieved (valid response rate: 99.6\%). Data from the 1046 valid questionnaires were analyzed.

\section{Questionnaire}

Every participant attended a face-to-face interview with well-trained investigators and completed a questionnaire designed based on the Questionnaire of Key Population's Smoking Behavior published by the Chinese Center for Disease Control and Prevention [19]. The current questionnaire included the participants' sociodemographic data, smoking status and knowledge, attitudes and practices in smoking control.

The questionnaire used in the present study had acceptable levels of reliability and validity. The internal consistency reliability test showed that the Cronbach's alpha coefficient was 0.85 . The construct validity test showed that the Kaiser-Meyer-Olkin was 0.84, and Bartlett's test of sphericity produced a result of $X^{2}$ value $=$ $8908.7(P<0.01)$.

\section{Statistical analysis}

Pearson's $x^{2}$ test and Fisher's exact probability method were used to compare the distribution of the categorical variables between/among groups. Statistical analyses were performed by Stata version 13.1. All reported probabilities ( $P$ values) were two sided. $P<0.05$ was considered statistically significant.

\section{Results}

\section{Demographic characteristics of participants}

Table 1 shows the demographic characteristics of the participants. Among the participants, $61.2 \%$ were male and $38.8 \%$ were female. Approximately $46.9 \%$ of the participants were aged between 31 and 40 years. Physicians, surgeons and pediatricians accounted for 32.7, 24.8 and $10.8 \%$ of the participants, respectively. Among the participants, $14.7 \%$ were current smokers.

\section{Participants' knowledge of smoking}

The participants' knowledge of smoking is summarized in Table 2. Among the participants, $81.4 \%$ agreed that smoking addiction is a cluster of cognitive, behavioral and physiological symptoms. In addition, $50.3 \%$ of the participants had heard that there are smoking cessation drugs. Approximately $46.9 \%$ of the participants responded to the question "Filter tips can decrease the harm from smoking" with "Agree". Approximately 59.2\% of the participants answered the question "Low-tar and low-nicotine cigarettes are as harmful to health as common cigarettes" with "Agree". The rates of responses to the above four items with "Agree" or "Yes" were significantly different among the age groups, professional title groups and department groups, as well as between smoking status groups. Among the five doctor department groups, pediatricians and surgeons presented the highest and lowest proportions of responses to the above four items with "Agree", respectively.

\section{Participants' knowledge of smoking-related diseases}

Participants' knowledge of smoking-related diseases is shown in Table 3. The participants who agreed that "smoking is related to lung cancer" and "smoking is related to coronary disease" accounted for 98.2 and $92.8 \%$ of the participants, respectively. The percentages of participants who responded to the questions "Smoking is related to osteoporosis" and "Smoking is related to male sexual dysfunction" with "Agree" were 56.2 and 60.9\%, respectively. The rates of responses to the above four items with "Agree" were significantly different among the five department groups. In addition, the pediatrician group had the highest proportion of answers to three questions "Smoking is related to coronary disease", "Smoking is related to osteoporosis" and "Smoking is related to male sexual dysfunction") of "Agree" among the five doctor department groups.

\section{Participants' attitudes towards smoking control}

Participants' attitudes towards smoking control are shown in Table 4. Among the participants, $81.9 \%$ agreed that doctors should be the role model of nonsmoking; 69.0\% answered the question "The doctor is the best role to persuade smokers to quit" with "Agree"; $72.0 \%$ responded to the question "Doctors should provide smoking cessation assistance actively" with "Agree"; and $58.1 \%$ agreed that "Provision of smoking cessation assistance is the doctor's responsibility". The rates of responses to the above four items with "Agree" were significantly different among the age groups, professional title groups and department groups, as well as between smoking status groups. Among the five doctor department groups, pediatricians and surgeons presented the 
Table 1 Demographic characteristics of participants

\begin{tabular}{|c|c|c|c|}
\hline Variable & Subgroup & Frequency & Percentage (\%) \\
\hline \multirow[t]{3}{*}{ Hospital } & Weifang People's Hospital & 344 & 32.89 \\
\hline & Liaocheng People's Hospital & 624 & 59.66 \\
\hline & Liaocheng Traditional Chinese Medicine Hospital & 78 & 7.46 \\
\hline \multirow[t]{2}{*}{ Sex } & 1.male & 640 & 61.19 \\
\hline & 2.female & 406 & 38.81 \\
\hline \multirow[t]{4}{*}{ Age(years) } & $\leq 30$ & 240 & 22.94 \\
\hline & $\leq 40$ & 491 & 46.94 \\
\hline & $\leq 50$ & 225 & 21.51 \\
\hline & $>50$ & 90 & 8.60 \\
\hline \multirow[t]{2}{*}{ Education } & Bachelor's degree or below & 327 & 31.26 \\
\hline & Graduate degree & 719 & 68.74 \\
\hline \multirow[t]{5}{*}{ Professional title } & No professional title & 90 & 8.60 \\
\hline & Primary professional title & 243 & 23.23 \\
\hline & Middle professional title & 290 & 27.72 \\
\hline & Deputy senior professional title & 262 & 25.05 \\
\hline & Senior professional title & 161 & 15.39 \\
\hline \multirow[t]{5}{*}{ Department } & Physician & 342 & 32.70 \\
\hline & Surgeon & 259 & 24.76 \\
\hline & Obstetrics and gynecology & 78 & 7.46 \\
\hline & Pediatrics & 113 & 10.80 \\
\hline & Other departments & 254 & 24.28 \\
\hline \multirow[t]{2}{*}{ Smoking status } & Smoker & 154 & 14.72 \\
\hline & Nonsmoker & 892 & 85.28 \\
\hline
\end{tabular}

highest and lowest proportions of responses to the above four items with "Agree", respectively.

\section{Participants' smoking control practices}

Participants' smoking control practices are summarized in Table 5. Among the participants, $85.2 \%$ often asked actively about the smoking history of patients or the patient's family members. Approximately $67.3 \%$ of participants would often educate the patients about the harms of smoking and persuade the patients to quit smoking if the patients had the habit of smoking. Only $4.9 \%$ of the participants had prescribed smoking cessation drugs for patients. There were significant differences in the above three items among the professional title groups and department groups. Pediatricians presented the highest proportions of participants who often asked about the smoking history of the patients or the patient's family members, educated the patients about the harm of smoking, persuaded the patients to quit smoking, and had prescribed smoking cessation drugs for patients.

\section{Discussion}

In the current study, participants did not have sufficient (response rate for "Agree": 45-60\%) common knowledge of smoking-related harms (such as filter tips and low-tar and low-nicotine cigarettes). In contrast, participants had better (response rate for "Agree": up to 80\%) specific knowledge of the smoking-related harms that were associated with medical conditions (for example, "smoking addiction is a cluster of cognitive, behavioral and physiological symptoms"). The possible reason for this difference might be related to doctors' educational experience. Specific knowledge of the smoking-related harms associated with medical conditions can be learned in medical universities but the most common knowledge of smoking-related harms is learned in special training on smoking control. However, most participants did not receive special training on smoking control, which suggested a shortage of special training on smoking control for Chinese doctors and other countries' doctors [2022]. As a result, doctors had little chance to learn the common knowledge of smoking-related harm. In conclusion, special training on smoking control is needed for doctors.

Participants were knowledgeable about the association between smoking and lung disease and coronary disease. However, they were not familiar with other uncommon smoking-related diseases (such as osteoporosis and male 
Table 2 Participants' cognition of smoking

\begin{tabular}{|c|c|c|c|c|c|c|c|c|c|c|c|c|c|}
\hline \multirow[t]{2}{*}{ Variables } & & \multicolumn{3}{|c|}{$\begin{array}{l}\text { Smoking addiction is a } \\
\text { cluster of cognitive, } \\
\text { behavioral and } \\
\text { physiological symptoms }\end{array}$} & \multicolumn{3}{|c|}{ Smoking cessation drugs } & \multicolumn{3}{|c|}{$\begin{array}{l}\text { Filter tips can decrease the } \\
\text { harm from smoking }\end{array}$} & \multicolumn{3}{|c|}{$\begin{array}{l}\text { Low-tar and low-nicotine } \\
\text { cigarettes are as harmful to } \\
\text { health as common } \\
\text { cigarettes }\end{array}$} \\
\hline & & Agree & $\begin{array}{l}\text { Do not } \\
\text { agree }\end{array}$ & $P$ & $\begin{array}{l}\text { Have } \\
\text { heard }\end{array}$ & $\begin{array}{l}\text { Do not } \\
\text { know }\end{array}$ & $P$ & Agree & $\begin{array}{l}\text { Do not } \\
\text { agree }\end{array}$ & $P$ & Agree & $\begin{array}{l}\text { Do not } \\
\text { agree }\end{array}$ & $P$ \\
\hline Total number & & $\begin{array}{l}851 \\
81.36\end{array}$ & $195,18.64$ & & $526,50.29$ & $520,49.71$ & & $\begin{array}{l}491 \\
46.94\end{array}$ & $555,53.06$ & & $\begin{array}{l}619 \\
59.18\end{array}$ & $427,40.82$ & \\
\hline \multirow[t]{2}{*}{ Sex } & 1 & $\begin{array}{l}519 \\
81.09\end{array}$ & $121,18.91$ & 0.78 & $315,49.22$ & $325,50.78$ & 0.39 & $\begin{array}{l}280 \\
43.75\end{array}$ & $360,56.25$ & $<0.01$ & $\begin{array}{l}355 \\
55.47\end{array}$ & $285,44.53$ & $<0.01$ \\
\hline & 2 & $\begin{array}{l}332 \\
81.77\end{array}$ & $195,18.64$ & & $211,51.97$ & $195,48.03$ & & $\begin{array}{l}211 \\
51.97\end{array}$ & $195,48.03$ & & $\begin{array}{l}264 \\
65.02\end{array}$ & $142,34.98$ & \\
\hline \multirow[t]{4}{*}{$\begin{array}{l}\text { Age } \\
\text { (years) }\end{array}$} & $\leq 30$ & $\begin{array}{l}186 \\
77.50\end{array}$ & $54,22.50$ & $<0.01$ & $98,40.83$ & $142,59.17$ & $<0.01$ & $\begin{array}{l}92, \\
38.33\end{array}$ & $148,61.67$ & $<0.01$ & $\begin{array}{l}129 \\
53.75\end{array}$ & $111,46.25$ & $<0.01$ \\
\hline & $\leq 40$ & $\begin{array}{l}390 \\
79.43\end{array}$ & $101,20.57$ & & $238,48.47$ & $253,51.53$ & & $\begin{array}{l}199 \\
40.53\end{array}$ & $292,59.47$ & & $\begin{array}{l}276 \\
56.21\end{array}$ & $215,43.79$ & \\
\hline & $\leq 50$ & $\begin{array}{l}189 \\
84.00\end{array}$ & $36,16.00$ & & $126,56.00$ & $99,44.00$ & & $\begin{array}{l}130 \\
57.78\end{array}$ & $95,42.22$ & & $\begin{array}{l}142 \\
63.11\end{array}$ & $83,36.89$ & \\
\hline & $\begin{array}{l}> \\
50\end{array}$ & $\begin{array}{l}86, \\
95.56\end{array}$ & $4,4.44$ & & $64,71.11$ & $26,28.89$ & & $\begin{array}{l}70, \\
77.78\end{array}$ & $20,22.22$ & & $\begin{array}{l}72 \\
80.00\end{array}$ & $18,20.00$ & \\
\hline \multirow[t]{2}{*}{ Education } & 1 & $\begin{array}{l}257 \\
78.59\end{array}$ & $70,21.41$ & 0.12 & $136,41.59$ & $191,58.41$ & $<0.01$ & $\begin{array}{l}138 \\
42.20\end{array}$ & $189,57.80$ & 0.04 & $\begin{array}{l}187 \\
57.19\end{array}$ & $140,42.81$ & 0.38 \\
\hline & 2 & $\begin{array}{l}594 \\
82.61\end{array}$ & $125,17.39$ & & $390,54.24$ & $329,45.76$ & & $\begin{array}{l}353 \\
49.10\end{array}$ & $366,50.90$ & & $\begin{array}{l}432 \\
60.08\end{array}$ & $287,39.92$ & \\
\hline \multirow[t]{5}{*}{$\begin{array}{l}\text { Professional } \\
\text { title }\end{array}$} & 1 & $\begin{array}{l}58 \\
64.44\end{array}$ & $32,35.56$ & $<0.01$ & $30,33.33$ & $60,66.67$ & $<0.01$ & $\begin{array}{l}20, \\
22.22\end{array}$ & $70,77.78$ & $<0.01$ & $\begin{array}{l}35 \\
38.89\end{array}$ & $55,61.11$ & $<0.01$ \\
\hline & 2 & $\begin{array}{l}192 \\
79.01\end{array}$ & $51,20.99$ & & $103,42.39$ & $140,57.61$ & & $\begin{array}{l}83 \\
34.16\end{array}$ & $160,65.84$ & & $\begin{array}{l}112 \\
46.09\end{array}$ & $131,53.91$ & \\
\hline & 3 & $\begin{array}{l}246 \\
84.83\end{array}$ & $44,15.17$ & & $144,49.66$ & $146,50.34$ & & $\begin{array}{l}132 \\
45.52\end{array}$ & $158,54.48$ & & $\begin{array}{l}181 \\
62.41\end{array}$ & $109,37.59$ & \\
\hline & 4 & $\begin{array}{l}213 \\
81.30\end{array}$ & $49,18.70$ & & $145,55.34$ & $117,44.66$ & & $\begin{array}{l}156 \\
59.54\end{array}$ & $106,40.46$ & & $\begin{array}{l}177 \\
67.56\end{array}$ & $85,32.44$ & \\
\hline & 5 & $\begin{array}{l}142, \\
88.20\end{array}$ & $19,11.80$ & & $104,64.60$ & $57,35.40$ & & $\begin{array}{l}100, \\
62.11\end{array}$ & $61,37.89$ & & $\begin{array}{l}114, \\
70.81\end{array}$ & $47,29.19$ & \\
\hline \multirow[t]{5}{*}{ Department } & 1 & $\begin{array}{l}277 \\
80.99\end{array}$ & $65,19.01$ & $<0.01$ & $150,43.86$ & $192,56.14$ & $<0.01$ & $\begin{array}{l}102 \\
29.82\end{array}$ & $240,70.18$ & $<0.01$ & $\begin{array}{l}161 \\
47.08\end{array}$ & $181,52.92$ & $<0.01$ \\
\hline & 2 & $\begin{array}{l}186 \\
71.81\end{array}$ & $73,28.19$ & & $88,33.98$ & $171,66.02$ & & $\begin{array}{l}89 \\
34.36\end{array}$ & $170,65.64$ & & $\begin{array}{l}121 \\
46.72\end{array}$ & $138,53.28$ & \\
\hline & 3 & $\begin{array}{l}67 \\
85.90\end{array}$ & $11,14.10$ & & $39,50.00$ & $39,50.00$ & & $\begin{array}{l}39, \\
50.00\end{array}$ & $39,50.00$ & & $\begin{array}{l}59, \\
75.64\end{array}$ & $19,24.36$ & \\
\hline & 4 & $\begin{array}{l}112, \\
99.12\end{array}$ & $1,0.88$ & & $100,88.50$ & $13,11.50$ & & $\begin{array}{l}107 \\
94.69\end{array}$ & $6,5.31$ & & $\begin{array}{l}105 \\
92.92\end{array}$ & $8,7.08$ & \\
\hline & 5 & $\begin{array}{l}209 \\
82.28\end{array}$ & $45,17.72$ & & $149,58.66$ & $105,41.34$ & & $\begin{array}{l}154 \\
60.63\end{array}$ & $100,39.37$ & & $\begin{array}{l}173 \\
68.11\end{array}$ & $81,31.89$ & \\
\hline \multirow[t]{2}{*}{$\begin{array}{l}\text { Smoking } \\
\text { status }\end{array}$} & 1 & $\begin{array}{l}115 \\
74.68\end{array}$ & $39,25.32$ & 0.02 & $65,42.21$ & $89,57.79$ & 0.03 & $\begin{array}{l}42, \\
27.27\end{array}$ & $112,72.73$ & $<0.01$ & $\begin{array}{l}67 \\
43.51\end{array}$ & $87,56.49$ & $<0.01$ \\
\hline & 2 & $\begin{array}{l}736 \\
82.51\end{array}$ & $156,17.49$ & & $461,51.68$ & $431,48.32$ & & $\begin{array}{l}449 \\
50.34\end{array}$ & $443,49.66$ & & $\begin{array}{l}552 \\
61.88\end{array}$ & $340,38.12$ & \\
\hline
\end{tabular}

Data are presented as frequency and percentage. Statistical analysis method: Pearson's $x^{2}$ test. Sex: 1: male, 2: female; Education: 1: bachelor's degree or below, 2: graduate degree; Professional title: 1: no professional title, 2: primary professional title, 3: middle professional title, 4: deputy senior professional title, 5: senior professional title; Department: 1: physician, 2: surgeon, 3: obstetrics and gynecology, 4: pediatrics; 5: other departments; Smoking status: 1: current smokers, 2: nonsmokers

sexual dysfunction). This result was similar to a previous Chinese study [10], supporting that knowledge of the harm from smoking was still insufficient among Chinese doctors. Knowledge determines attitude and action. A previous study showed that scientific knowledge about smoking control was important for doctors to contribute 
Table 3 Participants' cognition of smoking-related diseases

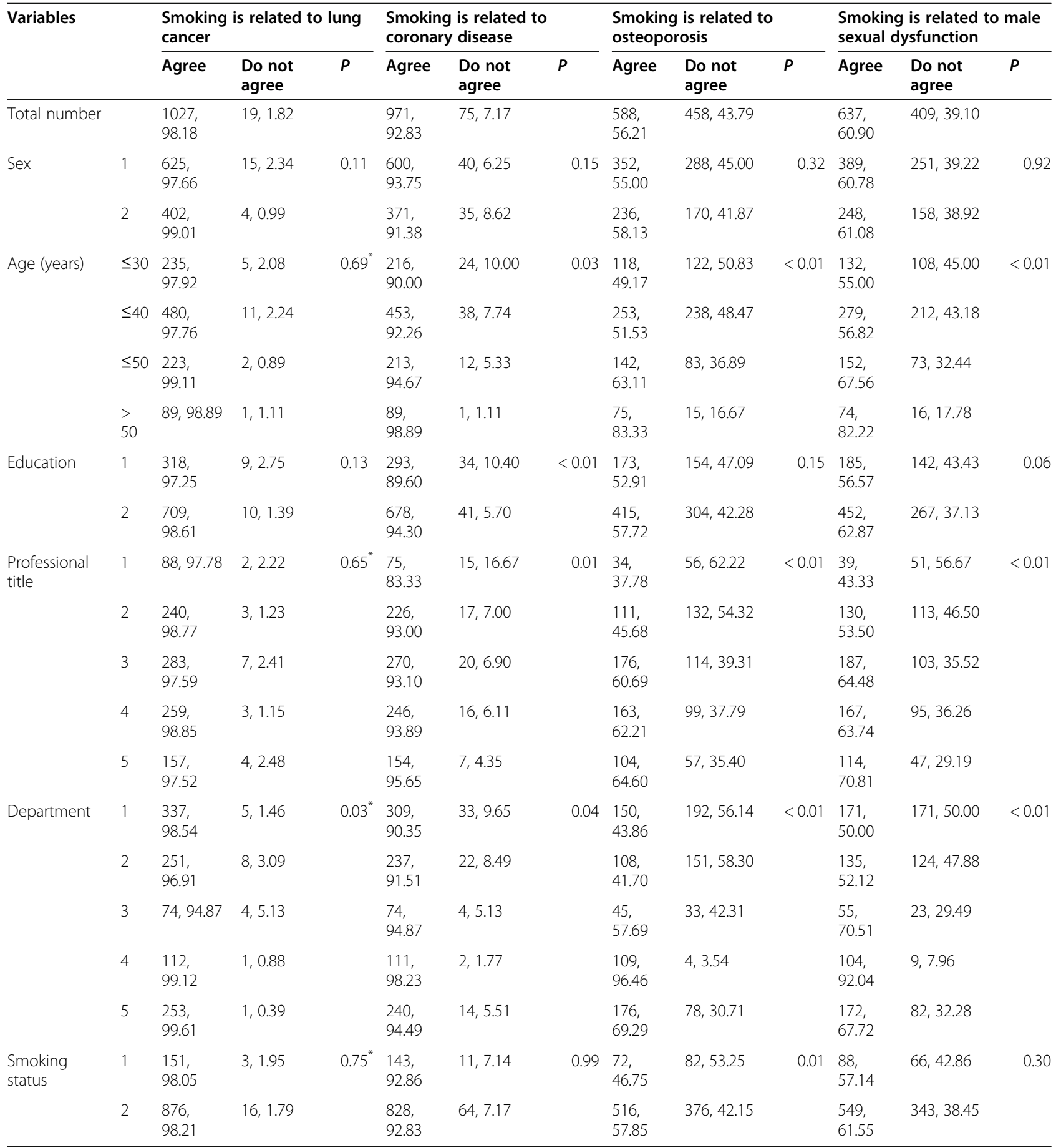

Data are presented as frequency and percentage. Statistical analysis method: ${ }^{*}$ is Fisher's exact probability method and others are Pearson's $x^{2}$ test. Sex: $1:$ male, 2 : female; Education: 1: bachelor's degree or below, 2: graduate degree; Professional title: 1: no professional title, 2: primary professional title, 3: middle professional title, 4: deputy senior professional title, 5: senior professional title; Department: 1: physician, 2: surgeon, 3: obstetrics and gynecology, 4: pediatrics; 5: other departments; Smoking status: 1: current smokers, 2: nonsmokers

to smoking control [10]. Therefore, doctors need more special training on smoking control.

In this study, most participants (approximately 80\%) realized their key role in smoking control, which was similar to the results of previous studies conducted in
China and other countries [7, 23, 24]. However, approximately $60 \%$ of participants believed that providing smoking cessation assistance was their responsibility, which was also consistent with the findings of previous studies $[7,20]$. There were some possible explanations for the 
Table 4 Participants' attitudes about smoking

\begin{tabular}{|c|c|c|c|c|c|c|c|c|c|c|c|c|c|}
\hline \multirow{2}{*}{\multicolumn{2}{|c|}{ Variables }} & \multicolumn{3}{|c|}{$\begin{array}{l}\text { Doctor should be the role } \\
\text { model of nonsmoking }\end{array}$} & \multicolumn{3}{|c|}{$\begin{array}{l}\text { Doctor is the best role to } \\
\text { persuade smokers to quit }\end{array}$} & \multicolumn{3}{|c|}{$\begin{array}{l}\text { Doctor should provide } \\
\text { smoking cessation assistance } \\
\text { actively }\end{array}$} & \multicolumn{3}{|c|}{$\begin{array}{l}\text { Provision of smoking } \\
\text { cessation assistance is } \\
\text { doctor's responsibility }\end{array}$} \\
\hline & & Agree & $\begin{array}{l}\text { Do not } \\
\text { agree }\end{array}$ & $P$ & Agree & $\begin{array}{l}\text { Do not } \\
\text { agree }\end{array}$ & $P$ & Agree & $\begin{array}{l}\text { Do not } \\
\text { agree }\end{array}$ & $P$ & Agree & $\begin{array}{l}\text { Do not } \\
\text { agree }\end{array}$ & $P$ \\
\hline Total number & & $\begin{array}{l}857 \\
81.93\end{array}$ & $189,18.07$ & & $\begin{array}{l}722 \\
69.02\end{array}$ & $324,30.98$ & & $\begin{array}{l}753 \\
71.99\end{array}$ & $293,28.01$ & & $\begin{array}{l}608 \\
58.13\end{array}$ & $438,41.87$ & \\
\hline \multirow[t]{2}{*}{ Sex } & 1 & $\begin{array}{l}510 \\
79.69\end{array}$ & $130,20.31$ & 0.02 & $\begin{array}{l}427 \\
66.72\end{array}$ & $213,33.28$ & 0.04 & $\begin{array}{l}438 \\
68.44\end{array}$ & $202,31.56$ & $<0.01$ & $\begin{array}{l}362 \\
56.56\end{array}$ & $278,43.44$ & 0.20 \\
\hline & 2 & $\begin{array}{l}347 \\
85.47\end{array}$ & $59,14.53$ & & $\begin{array}{l}295 \\
72.66\end{array}$ & $111,27.34$ & & $\begin{array}{l}315 \\
77.59\end{array}$ & $91,22.41$ & & $\begin{array}{l}246 \\
60.59\end{array}$ & $160,39.41$ & \\
\hline \multirow[t]{4}{*}{ Age (years) } & $\leq 30$ & $\begin{array}{l}186 \\
77.50\end{array}$ & $54,22.50$ & $<0.01$ & $\begin{array}{l}150 \\
62.50\end{array}$ & $90,37.50$ & $<0.01$ & $\begin{array}{l}161 \\
67.08\end{array}$ & $79,32.92$ & $<0.01$ & $\begin{array}{l}127 \\
52.92\end{array}$ & $113,47.08$ & $<0.01$ \\
\hline & $\leq 40$ & $\begin{array}{l}397 \\
80.86\end{array}$ & $94,19.14$ & & $\begin{array}{l}318 \\
64.77\end{array}$ & $173,35.23$ & & $\begin{array}{l}336 \\
68.43\end{array}$ & $155,31.57$ & & $\begin{array}{l}257 \\
52.34\end{array}$ & $234,47.66$ & \\
\hline & $\leq 50$ & $\begin{array}{l}188 \\
83.56\end{array}$ & $37,16.44$ & & $\begin{array}{l}171 \\
76.00\end{array}$ & $54,24.00$ & & $\begin{array}{l}172 \\
76.44\end{array}$ & $53,23.56$ & & $\begin{array}{l}149 \\
66.22\end{array}$ & $76,33.78$ & \\
\hline & $\begin{array}{l}> \\
50\end{array}$ & $86,95.56$ & $4,4.44$ & & $\begin{array}{l}83 \\
92.22\end{array}$ & $7,7.78$ & & $84,93.33$ & $6,6.67$ & & $\begin{array}{l}75 \\
83.33\end{array}$ & $15,16.67$ & \\
\hline \multirow[t]{2}{*}{ Education } & 1 & $\begin{array}{l}266 \\
81.35\end{array}$ & $61,18.65$ & 0.74 & $\begin{array}{l}201 \\
61.47\end{array}$ & $126,38.53$ & $<0.01$ & $\begin{array}{l}230 \\
70.34\end{array}$ & $97,29.66$ & 0.42 & $\begin{array}{l}175 \\
53.52\end{array}$ & $152,46.48$ & 0.04 \\
\hline & 2 & $\begin{array}{l}591 \\
82.20\end{array}$ & $128,17.80$ & & $\begin{array}{l}521 \\
72.46\end{array}$ & $198,27.54$ & & $\begin{array}{l}523 \\
72.74\end{array}$ & $196,27.26$ & & $\begin{array}{l}433 \\
60.22\end{array}$ & $286,39.78$ & \\
\hline \multirow[t]{5}{*}{$\begin{array}{l}\text { Professional } \\
\text { title }\end{array}$} & 1 & $59,65.56$ & $31,34.44$ & $<0.01$ & $\begin{array}{l}37 \\
41.11\end{array}$ & $53,58.89$ & $<0.01$ & $49,54.44$ & $41,45.56$ & $<0.01$ & $\begin{array}{l}26 \\
28.89\end{array}$ & $64,71.11$ & $<0.01$ \\
\hline & 2 & $\begin{array}{l}189 \\
77.78\end{array}$ & $54,22.22$ & & $\begin{array}{l}154 \\
63.37\end{array}$ & $89,36.63$ & & $\begin{array}{l}144 \\
59.26\end{array}$ & $99,40.74$ & & $\begin{array}{l}127 \\
52.26\end{array}$ & $116,47.74$ & \\
\hline & 3 & $\begin{array}{l}241 \\
83.10\end{array}$ & $49,16.90$ & & $\begin{array}{l}197 \\
67.93\end{array}$ & $93,32.07$ & & $\begin{array}{l}219 \\
75.52\end{array}$ & $71,24.48$ & & $\begin{array}{l}179 \\
61.72\end{array}$ & $111,38.28$ & \\
\hline & 4 & $\begin{array}{l}223 \\
85.11\end{array}$ & $39,14.89$ & & $\begin{array}{l}201 \\
76.72\end{array}$ & $61,23.28$ & & $\begin{array}{l}204 \\
77.86\end{array}$ & $58,22.14$ & & $\begin{array}{l}160 \\
61.07\end{array}$ & $102,38.93$ & \\
\hline & 5 & $\begin{array}{l}145 \\
90.06\end{array}$ & $16,9.94$ & & $\begin{array}{l}133 \\
82.61\end{array}$ & $28,17.39$ & & $\begin{array}{l}137 \\
85.09\end{array}$ & $24,14.91$ & & $\begin{array}{l}116 \\
72.05\end{array}$ & $45,27.95$ & \\
\hline \multirow[t]{5}{*}{ Department } & 1 & $\begin{array}{l}271 \\
79.24\end{array}$ & $71,20.76$ & $<0.01$ & $\begin{array}{l}221 \\
64.62\end{array}$ & $121,35.38$ & $<0.01$ & $\begin{array}{l}221 \\
64.62\end{array}$ & $121,35.38$ & $<0.01^{*}$ & $\begin{array}{l}171 \\
50.00\end{array}$ & $171,50.00$ & $<0.01$ \\
\hline & 2 & $\begin{array}{l}198 \\
76.45\end{array}$ & $61,23.55$ & & $\begin{array}{l}155 \\
59.85\end{array}$ & $104,40.15$ & & $\begin{array}{l}153 \\
59.07\end{array}$ & $106,40.93$ & & $\begin{array}{l}124 \\
47.88\end{array}$ & $135,52.12$ & \\
\hline & 3 & $74,94.87$ & $4,5.13$ & & $\begin{array}{l}74 \\
94.87\end{array}$ & $4,5.13$ & & $71,91.03$ & $7,8.97$ & & $\begin{array}{l}41 \\
52.56\end{array}$ & $37,47.44$ & \\
\hline & 4 & $\begin{array}{l}113 \\
100.00\end{array}$ & $0,0.00$ & & $\begin{array}{l}111 \\
98.23\end{array}$ & $2,1.77$ & & $\begin{array}{l}113 \\
100.00\end{array}$ & $0,0.00$ & & $\begin{array}{l}102 \\
90.27\end{array}$ & $11,9.73$ & \\
\hline & 5 & $\begin{array}{l}201 \\
79.13\end{array}$ & $53,20.87$ & & $\begin{array}{l}161 \\
63.39\end{array}$ & $93,36.61$ & & $\begin{array}{l}195 \\
76.77\end{array}$ & $59,23.23$ & & $\begin{array}{l}170 \\
66.93\end{array}$ & $84,33.07$ & \\
\hline \multirow[t]{2}{*}{$\begin{array}{l}\text { Smoking } \\
\text { status }\end{array}$} & 1 & $99,64.29$ & $55,35.71$ & $<0.01$ & $\begin{array}{l}82 \\
53.25\end{array}$ & $72,46.75$ & $<0.01$ & $84,54.55$ & $70,45.45$ & $<0.01$ & $\begin{array}{l}64 \\
41.56\end{array}$ & $90,58.44$ & $<0.01$ \\
\hline & 2 & $\begin{array}{l}758 \\
84.98\end{array}$ & $134,15.02$ & & $\begin{array}{l}640 \\
71.75\end{array}$ & $252,28.25$ & & $\begin{array}{l}669 \\
75.00\end{array}$ & $223,25.00$ & & $\begin{array}{l}544 \\
60.99\end{array}$ & $348,39.01$ & \\
\hline
\end{tabular}

Data are presented as frequency and percentage. Statistical analysis method: * is Fisher's exact probability method and others are Pearson's $X^{2}$ test. Sex: $1:$ male, 2 : female; Education: 1: bachelor's degree or below, 2: graduate degree; Professional title: 1: no professional title, 2: primary professional title, 3: middle professional title, 4: deputy senior professional title, 5: senior professional title; Department: 1: physician, 2: surgeon, 3: obstetrics and gynecology, 4: pediatrics; 5: other departments; Smoking status: 1: current smokers, 2: nonsmokers

difference between the responses of the two items. Firstly, although the WHO states that every health professional is responsible for asking about patients' tobacco use, assessing patients' willingness to quit smoking, advising patients to quit smoking, and further referring and arranging patients to participate in smoking cessation plans [25], doctors have not been required to provide smoking cessation assistance in China [26]. 
Table 5 Participants' actions about smoking

\begin{tabular}{|c|c|c|c|c|c|c|c|c|c|c|c|c|}
\hline \multirow{2}{*}{\multicolumn{2}{|c|}{ Variables }} & \multicolumn{4}{|c|}{$\begin{array}{l}\text { Ask about the smoking history of patients or the } \\
\text { patients' family members actively }\end{array}$} & \multicolumn{4}{|c|}{$\begin{array}{l}\text { Whether or not to educate the } \\
\text { patients about the harm of } \\
\text { smoking and persuade the patients } \\
\text { to quit smoking if the patients } \\
\text { have the habit of smoking }\end{array}$} & \multicolumn{3}{|c|}{$\begin{array}{l}\text { Have prescribed } \\
\text { smoking cessation drug } \\
\text { for patient }\end{array}$} \\
\hline & & Often & $\begin{array}{l}\text { When smoking is related to } \\
\text { disease }\end{array}$ & Rarely & $P$ & Often & Sometimes & Never & $P$ & Yes & No & $P$ \\
\hline Total number & & $\begin{array}{l}891 \\
85.18\end{array}$ & $91,8.70$ & $\begin{array}{l}64, \\
6.12\end{array}$ & & $\begin{array}{l}704 \\
67.30\end{array}$ & $323,30.88$ & $\begin{array}{l}19, \\
1.82\end{array}$ & & $\begin{array}{l}51 \\
4.88\end{array}$ & $\begin{array}{l}995 \\
95.12\end{array}$ & \\
\hline \multirow[t]{2}{*}{ Sex } & 1 & $\begin{array}{l}556, \\
86.88\end{array}$ & $53,8.28$ & $\begin{array}{l}31 \\
4.84\end{array}$ & 0.07 & $\begin{array}{l}427 \\
66.72\end{array}$ & $196,30.63$ & $\begin{array}{l}17, \\
2.66\end{array}$ & 0.04 & $\begin{array}{l}34 \\
5.31\end{array}$ & $\begin{array}{l}606 \\
94.69\end{array}$ & 0.41 \\
\hline & 2 & $\begin{array}{l}335 \\
82.51\end{array}$ & $38,9.36$ & $\begin{array}{l}33 \\
8.13\end{array}$ & & $\begin{array}{l}277 \\
68.23\end{array}$ & $127,31.28$ & $2,0.49$ & & $\begin{array}{l}17 \\
4.19\end{array}$ & $\begin{array}{l}389 \\
95.81\end{array}$ & \\
\hline \multirow[t]{4}{*}{ Age (years) } & $\leq 30$ & $\begin{array}{l}204 \\
85.00\end{array}$ & $14,5.83$ & $\begin{array}{l}22, \\
9.17\end{array}$ & 0.06 & $\begin{array}{l}154 \\
64.17\end{array}$ & $79,32.92$ & $7,2.92$ & $<0.01^{*}$ & $6,2.50$ & $\begin{array}{l}234 \\
97.50\end{array}$ & $<0.01$ \\
\hline & $\leq 40$ & $\begin{array}{l}421 \\
85.74\end{array}$ & $41,8.35$ & $\begin{array}{l}29 \\
5.91\end{array}$ & & $\begin{array}{l}309 \\
62.93\end{array}$ & $171,34.83$ & $\begin{array}{l}11 \\
2.24\end{array}$ & & $\begin{array}{l}18 \\
3.67\end{array}$ & $\begin{array}{l}473 \\
96.33\end{array}$ & \\
\hline & $\leq 50$ & $\begin{array}{l}191 \\
84.89\end{array}$ & $27,12.00$ & $7,3.11$ & & $\begin{array}{l}162 \\
72.00\end{array}$ & $62,27.56$ & $1,0.44$ & & $\begin{array}{l}10 \\
4.44\end{array}$ & $\begin{array}{l}215 \\
95.56\end{array}$ & \\
\hline & $\begin{array}{l}> \\
50\end{array}$ & $\begin{array}{l}75 \\
83.33\end{array}$ & $9,10.00$ & $6,6.67$ & & $\begin{array}{l}79 \\
87.78\end{array}$ & $11,12.22$ & $0,0.00$ & & $\begin{array}{l}17 \\
18.89\end{array}$ & $\begin{array}{l}73 \\
81.11\end{array}$ & \\
\hline \multirow[t]{2}{*}{ Education } & 1 & $\begin{array}{l}259 \\
79.20\end{array}$ & $37,11.31$ & $\begin{array}{l}31 \\
9.48\end{array}$ & $<0.01$ & $\begin{array}{l}212 \\
64.83\end{array}$ & $106,32.42$ & $9,2.75$ & 0.21 & $\begin{array}{l}19 \\
5.81\end{array}$ & $\begin{array}{l}308 \\
94.19\end{array}$ & 0.34 \\
\hline & 2 & $\begin{array}{l}632 \\
87.90\end{array}$ & $54,7.51$ & $\begin{array}{l}33 \\
4.59\end{array}$ & & $\begin{array}{l}492, \\
68.43\end{array}$ & $217,30.18$ & $\begin{array}{l}10 \\
1.39\end{array}$ & & $\begin{array}{l}32 \\
4.45\end{array}$ & $\begin{array}{l}687 \\
95.55\end{array}$ & \\
\hline \multirow[t]{5}{*}{$\begin{array}{l}\text { Professional } \\
\text { title }\end{array}$} & 1 & $\begin{array}{l}82, \\
91.11\end{array}$ & $1,1.11$ & $7,7.78$ & $<0.01$ & $\begin{array}{l}48 \\
53.33\end{array}$ & $38,42.22$ & $4,4.44$ & $<0.01^{*}$ & $3,3.33$ & $\begin{array}{l}87 \\
96.67\end{array}$ & $0.01^{*}$ \\
\hline & 2 & $\begin{array}{l}212 \\
87.24\end{array}$ & $12,4.94$ & $\begin{array}{l}19 \\
7.82\end{array}$ & & $\begin{array}{l}150 \\
61.73\end{array}$ & $87,35.80$ & $6,2.47$ & & $5,2.06$ & $\begin{array}{l}238 \\
97.94\end{array}$ & \\
\hline & 3 & $\begin{array}{l}248 \\
85.52\end{array}$ & $27,9.31$ & $\begin{array}{l}15 \\
5.17\end{array}$ & & $\begin{array}{l}196 \\
67.59\end{array}$ & $91,31.38$ & $3,1.03$ & & $\begin{array}{l}13 \\
4.48\end{array}$ & $\begin{array}{l}277 \\
95.52\end{array}$ & \\
\hline & 4 & $\begin{array}{l}224 \\
85.50\end{array}$ & $22,8.40$ & $\begin{array}{l}16 \\
6.11\end{array}$ & & $\begin{array}{l}182 \\
69.47\end{array}$ & $74,28.24$ & $6,2.29$ & & $\begin{array}{l}14 \\
5.34\end{array}$ & $\begin{array}{l}248 \\
94.66\end{array}$ & \\
\hline & 5 & $\begin{array}{l}125 \\
77.64\end{array}$ & $29,18.01$ & $7,4.35$ & & $\begin{array}{l}128 \\
79.50\end{array}$ & $33,20.50$ & $0,0.00$ & & $\begin{array}{l}16 \\
9.94\end{array}$ & $\begin{array}{l}145 \\
90.06\end{array}$ & \\
\hline \multirow[t]{5}{*}{ Department } & 1 & $\begin{array}{l}305 \\
89.18\end{array}$ & $25,7.31$ & $\begin{array}{l}12 \\
3.51\end{array}$ & $<0.01$ & $\begin{array}{l}247 \\
72.22\end{array}$ & $94,27.49$ & $1,0.29$ & $<0.01^{*}$ & $\begin{array}{l}11 \\
3.22\end{array}$ & $\begin{array}{l}331 \\
96.78\end{array}$ & $<0.01$ \\
\hline & 2 & $\begin{array}{l}218 \\
84.17\end{array}$ & $25,9.65$ & $\begin{array}{l}16 \\
6.18\end{array}$ & & $\begin{array}{l}143 \\
55.21\end{array}$ & $104,40.15$ & $\begin{array}{l}12 \\
4.63\end{array}$ & & $9,3.47$ & $\begin{array}{l}250 \\
96.53\end{array}$ & \\
\hline & 3 & $\begin{array}{l}53 \\
67.95\end{array}$ & $12,15.38$ & $\begin{array}{l}13 \\
16.67\end{array}$ & & $\begin{array}{l}61 \\
78.21\end{array}$ & $17,21.79$ & $0,0.00$ & & $1,1.28$ & $\begin{array}{l}77 \\
98.72\end{array}$ & \\
\hline & 4 & $\begin{array}{l}102 \\
90.27\end{array}$ & $4,3.54$ & $7,6.19$ & & $\begin{array}{l}102 \\
90.27\end{array}$ & $11,9.73$ & $0,0.00$ & & $\begin{array}{l}11 \\
9.73\end{array}$ & $\begin{array}{l}102 \\
90.27\end{array}$ & \\
\hline & 5 & $\begin{array}{l}213 \\
83.86\end{array}$ & $25,9.84$ & $\begin{array}{l}16 \\
6.30\end{array}$ & & $\begin{array}{l}151 \\
59.45\end{array}$ & $97,38.19$ & $6,2.36$ & & $\begin{array}{l}19 \\
7.48\end{array}$ & $\begin{array}{l}235 \\
92.52\end{array}$ & \\
\hline \multirow[t]{2}{*}{$\begin{array}{l}\text { Smoking } \\
\text { status }\end{array}$} & 1 & $\begin{array}{l}137 \\
88.96\end{array}$ & $9,5.84$ & $8,5.19$ & 0.33 & $\begin{array}{l}76 \\
49.35\end{array}$ & $71,46.10$ & $7,4.55$ & $<0.01^{*}$ & $\begin{array}{l}11 \\
7.14\end{array}$ & $\begin{array}{l}143 \\
92.86\end{array}$ & 0.16 \\
\hline & 2 & $\begin{array}{l}754 \\
84.53\end{array}$ & $82,9.19$ & $\begin{array}{l}56 \\
6.28\end{array}$ & & $\begin{array}{l}628 \\
70.40\end{array}$ & $252,28.25$ & $\begin{array}{l}12 \\
1.35\end{array}$ & & $\begin{array}{l}40 \\
4.48\end{array}$ & $\begin{array}{l}852 \\
95.12\end{array}$ & \\
\hline
\end{tabular}

Data are presented as frequency and percentage. Statistical analysis method: * is Fisher's exact probability method and others are Pearson's $\chi^{2}$ test. Sex: $1:$ male, 2 : female; Education: 1: bachelor's degree or below, 2: graduate degree; Professional title: 1: no professional title, 2: primary professional title, 3: middle professional title, 4: deputy senior professional title, 5: senior professional title; Department: 1: physician, 2: surgeon, 3: obstetrics and gynecology, 4: pediatrics; 5: other departments; Smoking status: 1: current smokers, 2: nonsmokers 
Secondly, most Chinese doctors thought that they were too busy to provide smoking cessation counseling [26], which also occurred in other countries [20].

The 5As method refers to 5 different counseling strategies for smoking cessation, including "Ask, Advise, Assess, Assist and Arrange" [12]. Previous similar studies in China found that approximately $50 \%$ of doctors often asked about the smoking history of the patients [24, 27]. It has been reported that approximately $28-70 \%$ of doctors ask patients about their smoking status in other countries (such as Belgium, Ireland, England and Egypt) [22, 23]. Similar to Mostafa's finding [23], the result of the current study (85.2\% of participants often asked the patients about their smoking status) was relatively satisfactory. Approximately $36 \%$ of health professionals always advised smokers to quit smoking in study conducted in 12 European countries [28]. The results of a study showed that $66 \%$ of participants advised their patients to quit smoking among obstetricians/gynecologists in Ohio [29]. In line with a previous study in China [30], approximately 67 and 30\% of doctors in this study often and sometimes persuaded the patients to quit smoking, respectively. The relatively acceptable behaviors of "Ask" and "Advise" in China may be attributed to Chinese tobacco control efforts from 2006 to the present, especially tobacco control education in hospitals and universities. With increasing age or professional title, an increasing number of participants asked about the patients' smoking history or advised the patients to quit smoking. In addition, greater proportions of doctors with graduate degrees often asked about the smoking history of patients. This situation reflected the effect of education about tobacco control in hospitals and universities to a certain degree. In the current study, only approximately $5 \%$ of participants provided assistance (prescribing smoking cessation drugs for patients), which was similar to results of previous Chinese studies [26, 31]. This proportion was lower than the average level of assistance reported by a systematic literature review (44\%) [13]. Approximately $15 \%$ of participants provided smoking cessation assistance among family physicians in Suez Canal University Hospitals [9]. There are some possible explanations for this difference in assistance between doctors in China and other countries. Firstly, few patients seek help for smoking cessation in Chinese hospitals. In China, offering cigarettes was considered a traditional social courtesy and a sign of respect [10]. On private occasions, the tradition of smoking has not changed fundamentally, and smoking is still treated as an individual freedom, which may result in the fact that few patients seek help for smoking cessation. Therefore, it is regarded as "offensive" and "harmful" for doctors to actively provide smoking cessation assistance [20]. Secondly, there is no requirement for doctors to actively provide smoking cessation assistance for smokers.

In this study, "Agree" response rates among pediatricians were higher than those among doctors in other departments for the majority of the items under study, which was similar to the findings of a United States study ( $83 \%$ of pediatricians would ask about the family members' smoking history) but different from the results of a Poland study (23\% of pediatricians would ask about the family members' smoking history) [32]. Due to children's physiological characteristics, children are more susceptible to adverse effects of smoke exposure [32]. Children's exposure to smoke will lead to a series of diseases (such as asthma, bronchitis, coughing, and pneumonia) [33]. Additionally, most couples currently have only one or two children in China, and children become more important to families. In this situation, Chinese pediatricians are required to learn more (including information about smoking and smoking-related diseases) and provide more information to parents. This may explain Chinese pediatricians' relatively high "Agree" response rates. "Agree" response rates of surgeons were lower than those of doctors in other departments for the majority of the items under study. This corresponded with the fact that the surgery department had the highest smoking rate in China among the different medical departments [34-36]. To reduce work-related stress and improve sociability [23], more surgeons have become smokers. Smoking doctors may pay less attention to knowledge of smoking, ignore the harms from smoking and be less likely to provide smoking cessation assistance $[10,23,37]$.

There were also some limitations in this study. Firstly, participants were not completely randomly sampled. To enroll participants conveniently and improve the feasibility of the study, purposive sampling was used to choose the sample hospital at the third step of sampling. This recruitment method may result in selection bias. We will use complete sampling to identify participants in further studies to overcome this limitation. Secondly, our questionnaire included the "Ask", "Advise" and "Assistance" of 5 As method but did not include "Assess" or "Arrange". Data from preinvestigation revealed that very few doctors provided "Assess" and "Arrange". For the sake of improving the feasibility of the study, questions about "Assess" and "Arrange" were not included. Therefore, it was difficult for the study to comprehensively evaluate doctors' practices regarding smoking control. Thirdly, the sample under study was not compared with the doctor population of China. It was unknown whether the demographic characteristics of the sample under study could precisely represent those of the doctor population 
of China. Therefore, care should be taken when generalizing the conclusions.

\section{Conclusions}

In conclusion, this study revealed that doctors in Shandong Province did not have sufficient knowledge of smoking control. Slightly more than half of doctors thought that providing smoking cessation assistance was their responsibility. Only a few doctors had prescribed smoking cessation drugs for their patients.

\section{Acknowledgments}

Not applicable

\section{Authors' contributions}

QW was responsible for analysis and interpretation of data, statistical analysis and writing the article. ZWW, SGF, XLZ, YL and CFZ were responsible for analysis and collection of data. CPW was responsible for creating the concept and design, supervising the study and obtaining funding. The authors read and approved the final manuscript.

\section{Funding}

This study was sponsored by the National Natural Science Foundation of China (Grant Number 71673202) and the Shandong Provincial Natural Science Foundation, China (Grant Number ZR014GL13). The funding bodies had no role in the design of the study; collection, analysis, and interpretation of data; or writing of the manuscript.

\section{Availability of data and materials}

The datasets used and/or analyzed during the current study are available from the corresponding author on reasonable request (chpwang@163.com).

\section{Ethics approval and consent to participate}

This study was approved by the Ethics Review Committee of Weifang Medical University. All participants included in the study provided their written informed consent prior to participation in the study.

\section{Consent for publication}

Not applicable

\section{Competing interests}

The authors declare that they have no competing interests.

\begin{abstract}
Author details
'Department of Epidemiology, Weifang Medical University, Weifang, Shandong, China. ${ }^{2}$ Department of Histology and Embryology, Weifang Medical University, Weifang, Shandong, China. ${ }^{3}$ Weifang people's hospital, Weifang, Shandong, China. ${ }^{4}$ School of Public health and Management, Weifang Medical University, Weifang, Shandong, China. ${ }^{5}$ Department of Environmental Health, Weifang Medical University, No. 7166 Baotong west street, Weifang 261053, Shandong, People's Republic of China.
\end{abstract}

Received: 15 May 2020 Accepted: 15 December 2020 Published online: 07 January 2021

\section{References}

1. WHO. The tobacco body. In. Geneva; 2019. p. 1.

2. $\mathrm{WHO}$. WHO global report on trends in prevalence of tobacco smoking 2000-2025, In. Edited by Second. Geneva; 2018. p. 11.

3. WHO. WHO global report on trends in prevalence of tobacco smoking 2000-2025, In. Edited by Second. Geneva; 2018. p. 6.

4. Chinese CDC. Reports of 2015 Chinese adult Tabaccl investigation. In. Beiling: 2016. p. 10

5. 2018 China Tobacco Control Report. http://www.chinacdc.cn/jkzt/sthd_3 844/slhd 4156/201905/t20190530_202932.html. Accessed 5 Jan 2020.

6. Chinese CDC. Chinese smoking control report-2013. In. BeiJing; 2013. p. 1.
7. Harutyunyan A, Abrahamyan A, Petrosyan V. Primary healthcare physicians' knowledge, attitude and practice towards smoking cessation in Armenia a qualitative study. In. Yerevan: American University of Armenia; 2015.

8. Han YW, Mohammad M, Liew SM. Effectiveness of a brief physician counselling session on improving smoking behaviour in the workplace. Asian Pac J Cancer Prev. 2014;15(17):7287-90.

9. Eldein HN, Mansour NM, Mohamed SF. Knowledge, attitude and practice of family physicians regarding smoking cessation counseling in family practice centers, Suez canal university, Egypt. J Family Med Prim Care. 2013;2(2):159-63.

10. Huang C, Guo C, Yu S, Feng Y, Song J, Eriksen M, Redmon P, Koplan J. Smoking behaviours and cessation services among male physicians in China: evidence from a structural equation model. Tob Control. 2013; 22(suppl 2):ii27-33.

11. Cantor SB, Deshmukh AA, Luca NS, Nogueras-Gonzalez GM, Rajan T, Prokhorov AV. Cost-effectiveness analysis of smoking-cessation counseling training for physicians and pharmacists. Addict Behav. 2015;45:79-86.

12. Petrides J, Collins P, Kowalski A, Sepede J, Vermeulen M. Lifestyle changes for disease prevention. Prim Care. 2019;46(1):1-12.

13. Bartsch AL, Harter M, Niedrich J, Brutt AL, Buchholz A. A systematic literature review of self-reported smoking cessation counseling by primary care physicians. PLoS One. 2016:11(12):e0168482.

14. ThinkTank Research Center for Health Development. A civil society perspective tobacco control in China-2018. In. BeiJing; 2016. p. 6-7.

15. China statistical yearbook 2018. http://www.stats.gov.cn/tjsj/ndsj/2018/ indexch.htm. Accessed 5 Jan 2020.

16. World Health Organization. Guidelines for Controlling and Monitoring the Tobacco Epidemic, Geneva: WHO. In. Luxembourg: WHO; 1998. p. 76.

17. Zhan S, Ye D, Tan H. Epidemiology. Beijing: People's medical publishing house; 2017.

18. Li Y, Chang $X$. Investigation and anaylysis of doctor's smoking status and its influence factors in Shenyang City. Mod Med J. 2015;43(2):169-72.

19. Qian Y, Li Y, Liu X. Survey on smoking among doctors and teachers in Beijing. Chin Prec Med. 2015;16(2):95-9.

20. Harutyunyan A, Abrahamyan A, Hayrumyan V, Petrosyan V. Perceived barriers of tobacco dependence treatment: a mixed-methods study among primary healthcare physicians in Armenia. Prim Health Care Res Dev. 2018:20.

21. Han H, Liu Y, Fu S, Li H, Zhu S, Jiang S. Physician smoking behaviors and their effect on smoking control counseling. Chin Gen Pract. 2014;17(11): 1217-20.

22. Matouq A, Khader Y, Khader A, Al-Rabadi A, Al Omari M, Iblan I, Al-Sheyab N. Knowledge, attitude, and behaviors of health professionals towards smoking cessation in primary healthcare settings. Transl Behav Med. 2018; 8(6):938-43.

23. Mostafa N, Momen M. Effect of physicians' smoking status on their knowledge, attitude, opinions and practices of smoking cessation in a university hospital, in Egypt. J Egypt Public Health Assoc. 2017;92(2):96-106.

24. Liu Y, Zhao B, Shi Y, Luo X. Investigation on the tobacco hazard perception and smoking cessation service of Yunnan physicians. Chin J Prev Contr Chron Dis. 2017:25(10):721-5.

25. WHO. The role of health professionals in tobacco. In. Geneva; 2005. p. 14.

26. Li $F$, Wang $X$, Dong $H$. Investigation and analysis on smoking status among doctors, teachers and civil servants. Chin J Health Educ. 2015; 31(9):846-59.

27. Su C, Li F, Liang J. Investigation about smoking of doctors, teachers and civil servants in Xiaodian county, Taiyuan city, China. Shanxi Med J. 2018; 47(21):2538-41.

28. Puska PM, Barrueco M, Roussos C, Hider A, Hogue S. The participation of health professionals in a smoking-cessation programme positively influences the smoking cessation advice given to patients. Int J Clin Pract. 2005;59(4):447-52.

29. TR J, JR D, JH P. Best practices for smoking cessation in pregnancy: do obstetrician/gynecologists use them in practice? J Women's Health (Larchmt). 2006;15(4):400-41.

30. Fu S, Liu Y, Sun X, Zhu S, Jiang S. Status of smoking and cessation services: analysis in Chinese doctors. Chin Gen Pract. 2014;17(11):1221-4.

31. Wu X, Yang Y, Jiang Y, Li Q, Feng G, Nan Y. Study on abilities of smoking cessation services and its associated factors among Chinese physicians. Chin J Prev Contr Chron Dis. 2010:18(4):346-9.

32. Ratajczak A, Ratajczak K, Feleszko W. A cross-sectional study of smoking behaviors and attitudes of parents in pediatric primary care settings. Int J Environ Res Public Health. 2018;15(7). 
33. Vitoria PD, Machado JC, Ravara SB, Araujo AC, Samorinha C, Antunes H, Rosas M, Becona E, Precioso J. Portuguese children's exposure to secondhand tobacco smoke in the family car. Gac Sanit. 2015;29(2):131-4.

34. Zhang J, Sun F, Qiu S, Yao H, Li X, Li D. Study on the status of smoking among doctors in Hefei. Chin J Dis Control Prev. 2010;14(3):272-4.

35. Liu J, Zhang Y, Wang Y, Fu C. Epidemiologic investigation into medical personnel smoking in Zhengzhou. Mod Prev Med. 2006;33(5):745-748,759.

36. Feng $L, X u C$. A survey on cigarette smoking related behavior among medical doctors in Changzhou. Chin Prev Med. 2013;14(11):852-6.

37. Reile R, Parna K. Do physicians address their patients' smoking behavior? Results from a nationwide survey among physicians in Estonia. Public Health. 2018;161:1-4.

\section{Publisher's Note}

Springer Nature remains neutral with regard to jurisdictional claims in published maps and institutional affiliations.

Ready to submit your research? Choose BMC and benefit from:

- fast, convenient online submission

- thorough peer review by experienced researchers in your field

- rapid publication on acceptance

- support for research data, including large and complex data types

- gold Open Access which fosters wider collaboration and increased citations

- maximum visibility for your research: over $100 \mathrm{M}$ website views per year

At $\mathrm{BMC}$, research is always in progress.

Learn more biomedcentral.com/submissions 